\title{
Potential targets for prevention and treatment of non-alcoholic fatty liver disease in adults
}

\author{
Jarnail S. Braich* \\ Department of Pharmacology, Pt BD Sharma Postgraduate Institute of Medical Sciences, Rohtak, Haryana, India
}

Received: 29 September 2020

Accepted: 30 October 2020

*Correspondence:

Dr. Jarnail Singh,

Email: drjarnailuhsr@gmail.com

Copyright: $(9$ the author(s), publisher and licensee Medip Academy. This is an open-access article distributed under the terms of the Creative Commons Attribution Non-Commercial License, which permits unrestricted non-commercial use, distribution, and reproduction in any medium, provided the original work is properly cited.

\begin{abstract}
Non-alcoholic fatty liver disease (NAFLD) is a common condition worldwide contributing to serious liver disorders like hepatic fibrosis and hepatocellular carcinoma. Most common of NAFLD etiology is a sedentary lifestyle and taking a high-calorie diet. Presently there is no drug available for the treatment of NAFLD and only dietary measures, increased physical activity and exercise, antioxidants are the options. In this review, detailed pathophysiology, diagnostic methods and potential targets for prevention and timely treatment of NAFLD have been described.
\end{abstract}

Keywords: Non-alcoholic fatty liver disease, Non-Alcoholic Steatohepatitis, Antioxidants, Hypolipidemic drugs, Insulin resistance, Insulin sensitizers

\section{INTRODUCTION}

Non-alcoholic fatty liver disease (NAFLD) is a common liver disorder, and it has become a silent epidemic affecting $25 \%-44 \%$ of the overall population. This liver disorder is common in patients with diabetes type 2 (T2DM) ${ }^{1}$ NAFLD can be divided into hepatic steatosis (HS), non-alcoholic steatohepatitis (NASH) and liver cirrhosis. It is estimated that $25 \%$ of the world population has NAFLD with highest prevalence rates reported from South America (31\%) and the middle east (32\%), Asian populations (27\%) and lowest from Africa (14\%). NAFLD, a component of metabolic syndrome (MetS), is defined as the presence of $\geq 5 \%$ hepatic steatosis (HS) in the absence of liver diseases such as chronic viral hepatitis, autoimmune hepatitis, hemochromatosis, Wilson's disease, use of drugs such as amiodarone, tamoxifen or significant alcohol consumption (>21 drinks/week in men and $>14$ drinks/week in women). ${ }^{2}$ NAFLD is omnipresent; some of these patients can develop NASH, which is defined histologically by the presence of HS with hepatocyte damage. Hepatic fibrosis
(HF) is the most crucial histological feature associated with mortality in NASH. NASH is a risk factor for cirrhosis in adults and the second indication for liver transplantation in the United States. ${ }^{3}$ Development of NAFLD is closely linked to lifestyle factors such as excessive intake of calorie-dense food and reduced physical activity and exercise. During the last 3 decades, there is a significant increase in body mass index (BMI) and prevalence of obesity which are pathophysiological drivers of NAFLD. Usually, NAFLD patients are obese with insulin resistance (IR) \pm type 2 diabetes (T2DM), dyslipidemia, hypertriglyceridemia, and hypertension (the risk factors for cardiovascular diseases). ${ }^{4}$ NAFLD is reported in $76 \%$ of patients with $\mathrm{T} 2 \mathrm{DM}$ and more than $90 \%$ of severely obese patients undergoing bariatric surgery. Because risk factors for NAFLD and CVD are common, cardiac-related death is one of the leading causes of death in NAFLD patients. Elevated liver enzymes underestimate the prevalence of NAFLD because up to $78 \%$ of NAFLD patients have only mildly elevated liver enzymes. ${ }^{4}$ 
Obesity was present in $51.34 \%$ NAFLD and $81.83 \%$ NASH patients and diabetes mellitus was seen in $23 \%$ NAFLD and 47\% NASH patients. Diabetes in NAFLD is a risk for progression to NASH, cirrhosis, and mortality. Prevalence of MetS was $42.54 \%$ in NAFLD and $70.65 \%$ in NASH with dyslipidemia patients. High prevalence of MetS and dyslipidemia in NAFLD patients suggests the requirement of aggressive treatment to control the risk of CVD. Although rare hepatocellular carcinoma (HCC) is a consequence of NAFLD, with much less incidence than with chronic hepatitis B, NAFLD and especially NASH patients have high liver-specific mortality and overall mortality. 4

\section{PATHOGENESIS AND DIAGNOSIS OF NAFLD}

Diagnosis of NAFLD requires clinicopathological correlation, excluding excessive consumption of alcohol and inflammation and liver injury confirmed by biopsy. Imaging techniques, ultrasonography (USG) and magnetic resonance imaging (MRI) are commonly used non-invasive alternatives with the sensitivity of $89 \%$ and $77 \%$, and specificity of $93 \%$ and $89 \%$ in diagnosing HS and NASH respectively. ${ }^{5}$ USG is subjective thus not suitable for assessing HS, while MRI is more objective and better for quantifying steatosis. Subtle histological changes of inflammation and ballooning cannot be detected, thus differentiate HS from NASH; therefore, liver biopsy is the "gold" standard tool to diagnose $\mathrm{NASH}$, although it is an invasive procedure. ${ }^{6}$ Recently several non-invasive diagnostic indices may be utilized to diagnose NAFLD and NASH, including fatty liver index (FLI), the hepatic steatosis index (HSI), and the ZJU (Zhejiang university) index. ${ }^{7}$ In patients presenting with fatty liver secondary causes of HS need to be excluded. Chronically raised serum liver enzymes may be due to viral hepatitis, autoimmune liver disease and drug hepatotoxicity. 5

Pathological findings based on liver biopsy are the gold standard for diagnosing and staging HS, fibrosis and cirrhosis. Inter-observer and intra-observer variation between histopathologists complicate the diagnosis of NASH by liver biopsy, fibrosis, lobular inflammation and ballooning. 5

Mostly, mild elevation of liver enzymes, alanine aminotransferase (ALT) and aspartate aminotransferase (AST) and gamma-glutamyl transferase (GGT) is the only finding in NAFLD patients. ${ }^{5}$ Levels of ALT are higher in NAFLD patients; in $80 \%$ of patients with fatty liver disease ALT levels are within normal limits. As fibrosis becomes advanced aminotransferase levels tend to fall and ALT:AST ratio may become reversed. Persons with NAFLD having high levels of ALT are more likely to have NASH, but normal levels of ALT does not exclude NASH and advanced fibrosis. Elevation in ALT and GGT is part of MetS, but liver enzyme levels have low sensitivity, and negative prediction of NAFLD and patient can ha have a histological spectrum of NAFLD but normal liver function tests (LFT). ${ }^{6}$ Levels of hepatic apoptosis are increased in NASH and can be assessed in plasma by measuring cytokeratin-18 (CK-18) fragments. CK-18 is an intra-hepatic intermediate filament protein cleaved by caspase enzymes during apoptosis. CK-18 fragment levels are raised in NASH patients compared with simple steatosis and fall with weight loss induced by bariatric surgery. Adiponectin, tumor necrosis factor (TNF) and interleukin-6 (IL-6) play a crucial role in mediating HS, insulin resistance (IR) and inflammation in NASH. ${ }^{7,8}$ Adiponectin, an insulin-sensitizing antiinflammatory adipocytokine, is reduced in IR states such as obesity and diabetes. Adiponectin has a negative correlation with the degree of HS in patients with NAFLD. TNF- $\alpha$, a major inflammatory hepatic cytokine, is also secreted by adipose tissue and antagonizes the effects of adiponectin. ${ }^{5}$ IL-6 is also implicated in the pathogenesis of IR; hepatic and systemic IL-6 levels are increased in patients with NASH. Adipocyte fatty-acid binding protein (AFBP), secreted by adipocytes and macrophages, is associated with IR and necroinflammation in NAFLD patients, but its exact role in the pathogenesis of NAFLD is not clear. Serum prolidase enzyme is involved in collagen breakdown, serum prolidase enzyme activity (SPEA) is a non-invasive marker of hepatic fibrosis (HF), but validation is required. ${ }^{5}$

Soluble receptor for advanced glycation end (sRAGE) products also generates hepatic inflammatory responses and IR. Plasma levels of sRAGE are readily measurable and are associated with various parameters of the MetS. ${ }^{9}$ Association between sRAGE and NASH is independent of age, gender, IR, BMI and liver enzyme levels.

Hepatic oxidative stress (OS) is a crucial factor for hepatocyte injury and development of NASH. Increase in fatty acid oxidation results in the production of free radicals and thereby, the production of lipid peroxidation products. ${ }^{5}$ Plasma malondialdehyde levels are raised in NAFLD compared to controls, but not correlated to necro-inflammatory activity. The markers of lipid peroxidation, thiobarbituric acid-reacting substance and oxidized low-density lipoprotein (LDL) are increased in the plasma in patients with NASH reflecting IR compared to age, gender, BMI matched controls. ${ }^{5}$ NAFLD has a complex pathophysiological association with CVD involving oxidative stress, atherogenic dyslipidemia, endothelial dysfunction, increased carotid intima-media thickness (IMT), IR and abnormal adipokine profile. NAFLD is also associated with a high incidence of colorectal adenomas and high grade colorectal carcinomas. $^{10}$

\section{MANAGEMENT OF NAFLD}

Currently, there is no effective pharmacotherapy for treatment for NAFLD. In most of the trials in NAFLD patients, there was some decrease in steatosis, inflammation and even ballooning but no evidence of a 
reduction in fibrosis. Either there is no right antifibrotic agent against NAFLD or design of the trial was not more than 2 years of intervention. NAFLD has a slow progressive nature; thus, longer treatment intervention trials are needed. The non-pharmacological approach is advised to all NAFLD patients, even HS, because NAFLD increases the risk for MetS, CVD and several malignancies. Screening for HCC should be carried out for all patients with hepatic cirrhosis and careful monitoring at regular screening programs for colorectal, prostate, breast and cervical cancer.

\section{Non-pharmacological treatment}

Exercise and weight loss: moderate exercise and weight loss are associated with improvement in insulin sensitivity overweight or obese NAFLD patients. Weight reduction may be achieved by dieting, physical activity, exercise, antiobesity drugs and bariatric surgery in morbid obesity. ${ }^{11}$ Exercise has beneficial effects on NAFLD and IR, even when weight loss is not there, but weight loss and exercise has a synergistic effect. Patients should perform aerobic and restrictive exercise $\geq 3-4$ times per week and $\geq 400$ calories per session. ${ }^{12}$ Weight loss of $3-5 \%$ in $\mathrm{HS}$ and $7-10 \%$ in NASH should be promoted in NAFLD patients, except for those rare NAFLD patients with average weight. ${ }^{2}$ Weight loss of at least $5 \%$ of body weight improves HS and IR, and weight loss by $7-9 \%$ improves inflammation, hepatocellular ballooning and NASH. To enhance the efficacy of weight loss programs, these should be associated with cognitivebehaviour therapy. ${ }^{13}$ Twenty-four exercise only studies have revealed that exercise produced $20-30 \%$ relative reduction in HS, independent of weight loss. ${ }^{3,7}$ Aerobic, resistance/strength training or high intensity interval training have a relatively similar effect on liver fat. ${ }^{4}$ Exercise training increases peroxisome proliferatoractivated receptor gamma coactivator 1-alpha (GGC-1 $\alpha$ ) expression, improves mitochondrial function and leads to reduced HS, inflammation, fibrosis and tumour necrosis. Optimal duration and intensity of physical exercise for the prevention and treatment of NAFLD have not been established. Exercise training, routinely recommended for treatment of NAFLD, is a robust approach for alleviating the pathogenesis of NAFLD, but further clinical and experimental studies are required. Exercise per se might independently ameliorate HS. Moderate exercise for 150$200 \mathrm{~min} /$ week, including aerobic exercise and resistance exercise is recommended. ${ }^{14}$

Energy expenditure is increased during exercise, promoting glucose and lipid metabolism, which ameliorates obesity and NAFLD. Exercise training decreases hepatic gluconeogenesis, hepatic capacity for gluconeogenesis, lactate transport capacity and oxidative capacity are increased by training. Exercise training also increases antioxidant enzymes (superoxide dismutase-1 (SOD-1), SOD-2, catalase (CAT) and glutathione peroxidase in the liver and oxidative damage is reduced. Antioxidant effect of training is a possible mechanism on
NAFLD characterized by HS, inflammation and oxidative damage. Exercise-induced protein secretion from the liver: Serum insulin-like growth factor-1 (IGF1) is lower in patients with diabetes and NAFLD than in healthy subjects, and negatively correlated with the severity of hepatic fibrosis in patients with NAFLD.

Diet should be hypocaloric adjusted for the need and bodyweight of the patient, constituting fat (n3-PUFA, advice 2-3 oily fish meals per week, $\leq 25 \%$ as MUFA, avoid SAF ( $<7 \%$ of total energy), cholesterol $\leq 200$ $\mathrm{mg} /$ day), carbohydrates $\geq 50 \%$ as whole grains avoid fructose from corn syrup, but no need to restrict coffee. The western dietary pattern, which combines high saturated fats with fructose intake, is implicated in the development of NAFLD. A low calorie-diet is compulsory is required to accomplish weight loss $(<1500$ $\mathrm{kcal} /$ day) Most studied protective diet in NAFLD is Mediterranean diet (MD). ${ }^{11,12}$

MD is a dietary pattern constituting increased intake of plant-based foods, whole grains, cereals, seeds, nuts, legumes, vegetables and fruits, moderate consumption of protein source foods (fish, seafood, and poultry), low to moderate red wine consumption, low consumption of meat, milk, dairy products and usual optimal physical activity. MD contains MUFA from olive oil, a greater omega-3/omega-6 ratio PUFA, polyphenols, carotenoids and high fibre foods. MetS patients getting MD show improvement in IR and inflammatory markers (CRP, IL6, IL-7, and IL-8). MD may modulate CVD risk factors, e.g. TC and LDLC. In NAFLD, MD decreases hepatic fat improves hepatic insulin sensitivity which is independent of exercise and weight loss. ${ }^{15}$ Because of moderate quality of evidence, MD diet has been recommended for the treatment of NASH by various authorities. ${ }^{16}$ Patients with dyslipidemia benefit from low-fat diets, and those with IR/T2DM from low carbohydrate diets. Lipids should be mainly polyunsaturated fatty acids (PUFA); mainly $\omega 3$ from fish oil, $25 \%$ of fat should be from mono-unsaturated fatty acids (MUFA).

Alcohol consumption higher than 2-3 drinks/day not only increases the risk of hepatic cirrhosis; it also increases the risk for overall death and some cancers. Low intake of alcohol decreases overall mortality by a decrease in cardiovascular events, less risk for diabetes and MetS. Some studies show a possible beneficial effect in NAFLD for very mild alcohol consumption. The benefit of mild alcohol consumption in obese is not known because obesity and alcohol are synergistic in promoting NAFLD and HCC. In patients with NASH and associated cirrhosis alcohol intake should be strongly discouraged because regular intake of alcohol increases the risk for HCC by more than 3 fold. ${ }^{17,18}$

\section{Pharmacotherapy}

There is no standard drug for the treatment of NAFLD. Dietary modification, weight loss and physical activity 
are the primary treatment line for NAFLD. Based on the multiple-hit pathogenesis, simultaneous multiple targetmanagement is proposed, instead of targeting only one of IR, obesity, dyslipidemia or oxidative stress. Currently, there is a limited experience for combination therapy in NAFLD. Treatment strategies for NAFLD include; identification and treatment of associated metabolic conditions, e.g. T2DM and dyslipidemia, improving IR by weight loss, exercise or pharmacotherapy and using hepatoprotective agents such as antioxidants.

MetS and central obesity, glucose intolerance, hypertriglyceridemia and hypertension are associated with CVD morbidity and mortality. Newly diagnosed NAFLD patients should be screened for these conditions, and appropriate treatment should be started to improve NAFLD and risk for CVD.

\section{Insulin sensitizing/antidiabetic drugs}

Association between NAFLD and IR is well established, playing an essential role in hepatic fat accumulation and its progression to NASH. Also, IR is predictive of necroinflammatory NAFLD and obesity; and T2DM is the crucial risk factor for severe liver disease. ${ }^{11}$ Insulin sensitizers, biguanides (metformin) and thiazolidinediones (TZDs), pioglitazone and rosiglitazone have been evaluated as a possible treatment for NAFLD. Several studies show beneficial effects of TZDs in NAFLD patients, improving transaminase levels and hepatic inflammation, but rosiglitazone has been banned because of significant adverse effects. ${ }^{19}$ Pioglitazone has been evaluated for its effectiveness in the treatment of NAFLD in controlled trials. Improvement in ALT (by $50 \%$ ), steatosis (by 54\%), insulin sensitivity (by 48\%), ballooning necrosis, hepatic inflammation but not fibrosis was observed in diet plus pioglitazone group versus diet plus placebo. NAFLD patients with T2DM have 2.6 higher risk for developing NASH compared with those without T2DM; the risk further increases in obese diabetics. Also, T2DM patients have 3-fold higher risk of developing HCC, thus increasing the risk of liver related death in NAFLD patients by up to 22 fold.${ }^{10}$ Liver disease is expected but not universal in T1DM but by "multiple hit" theory, other risk factors, e.g. obesity, poor glycemic control and age are associated with the development of NAFLD in T1DM patients. Long term safety of pioglitazone is not well established; it may be used only in biopsy proven NASH patients.

Metformin is effective in patients with NAFLD in several clinical trials. ${ }^{20}$ Metformin, a widely used in first-line treatment of T2DM, lowers blood glucose by decreasing gluconeogenesis in the liver, stimulating glucose uptake by muscle and increasing fatty acid oxidation in adipose tissue with final effect as improvement of peripheral insulin sensitivity. Beneficial effects of metformin are related to phosphorylation and nuclear export of liver kinase B1 (LKB1). LKB1 activates adenosine monophosphate-activated protein kinase (AMPK) which can stimulate ATP producing catabolic pathways such as glycolysis, fatty acid oxidation, and mitochondrial biogenesis and inhibit ATP consuming anabolic processes such as gluconeogenesis, glycogen, fatty acid and protein synthesis. Upon activation to stress in muscle AMPK induces hexokinase II expression and GLUT4 gene upregulation and translocation to cell membrane leading to an increase in glucose uptake. It also inhibits glycogen synthase thus decrease in glycogen synthesis. In the liver, AMPK reduces hepatic gluconeogenesis by inducing phosphorylation of CREB-binding protein (CBP) and thus dissociation of gluconeogenic CREBCBP-TORC2 transcriptional complex. ${ }^{20}$ Activation of AMPK inactivates ACC, and HMG-CoA reductase decreases fatty acid synthase (FAS) carboxylase expression and activates malonyl-CoA, finally leading to a decrease in fatty acid and cholesterol synthesis. AMPK also inhibits sterol regulatory element-binding protein-1c (SREB1c) which is a transcription factor for genes involved in the fatty acid synthesis. SREB-1c is induced by high glucose and insulin levels and is inappropriately increased in NAFLD patients. Recent studies show that metformin also modulates adipokine synthesis or secretion. Adiponectin, induced by metformin, directly stimulates AMPK and prevents hepatic fat accumulation by increasing $\beta$-oxidation of free fatty acids (FFA) and decreasing their de novo synthesis. ${ }^{21}$

Continuation of use of metformin after diagnosis of cirrhosis significantly improved survival in all stages of cirrhosis, which suggests that metformin is safe and welltolerated in patients with decompensated liver disease. ${ }^{22}$ In patients with NASH related cirrhosis metformin had a protective effect probably due to the pleiotropic effects of metformin in cell proliferation and differentiation, apoptosis and inflammation and metabolic pathways of glucose and lipid homeostasis. ${ }^{23}$

T2DM is the most common condition correlated with NAFLD, and there is a higher risk of inflammation and hepatic damage. Many drugs have been tested to improve HS or to avoid the progression to cirrhosis, with variable results. Many trials on antidiabetic medications, such as metformin, dipeptidyl peptidase-4 (DPP-4) inhibitors, SGLT-2 inhibitors or thiazolidinediones, showed an improvement in weight and liver enzymes, but data regarding histology lack and with no accordance. Insulin sensitizers seem to be the promising agents, because they can treat both NAFLD manifestations and diabetes, preventing worsening of hepatic damage. Because of no specific therapies for patients with NAFLD or NASH, it is to clarify the hepatic safety of antidiabetics.

All antidiabetic agents can be used in patients with compensated cirrhosis, but there are still no data available about patients with advanced cirrhosis, especially for glitazones, incretins, DPP-4 inhibitors and SGLT-2 inhibitors. $^{24}$ 


\section{Anti-oxidants}

Vitamin $\mathrm{E}$ is potent anti-oxidant in nature, with several dietary sources. The antioxidant property of $\alpha, \beta, y$ isoforms of tocotrienols and tocopherols is similar. Vitamin $\mathrm{E}$ has more significant antioxidant action on reactive nitrogen species (RNS) which include nitric oxide (NO), nitrogen dioxide $\left(\mathrm{NO}_{2}\right)$ and peroxynitrite $\left(\mathrm{ONOO}^{-}\right)$.Vitamin $\mathrm{E}$ shows pro-oxidant effects at high doses. ${ }^{25-27}$ Vitamin $\mathrm{E}$ has been used as monotherapy in clinical trials to treat NAFLD or NASH with histological and biochemical improvement in the liver. In these trials dose of vitamin E was 100-1200 IU/d and duration of study was 24 weeks to 2 years. Vitamin E shows beneficial histological and biochemical changes in the liver when used in combination with vitamin $\mathrm{C}$, ursodeoxycholic acid (UDCA) and atorvastatin. Longterm safety is a concern in patients with NASH. ${ }^{28} \mathrm{~A}$ meta-analysis has suggested that supplemental vitamin $\mathrm{E}$ in high dosage ( $\geq 400$ IU/day) may increase all-cause mortality. ${ }^{29}$ A meta-analysis on the effect of vitamin $E$ on the incidence of stroke has shown a higher relative risk of hemorrhagic stroke by $22 \%$, while the risk of ischemic stroke was reduced by $10 \% .{ }^{30}$ Vitamin $\mathrm{E}$ has also been associated with an increased risk of prostate cancer. ${ }^{31}$ Coffee has antioxidant, anti-inflammatory and antifibrotic properties; thus, there is an inverse association between caffeine intake and NASH. In a retrospective study, coffee consumption was recorded at baseline and 7 years in NAFLD patients; those were drinking coffee >3 cups/day developing HCC had a lower fibrosis score; thus it can reduce the risk of developing HCC, in those who consumed 3 or more cups/day had $44 \%$ lower less risk of $\mathrm{HCC}$, but further studies are required in this regard. ${ }^{32-34}$ Resveratrol, a dietary antioxidant found in red wine, appears to improve IR and OS. Extensive clinical trials have not been conducted in this regard. Some degree of regular alcohol consumption over a lifetime vs. the minimal intake appears to have a protective effect on histopathological severity. More studies are required for the recommendation of regular alcohol consumption recommendation in NAFLD. ${ }^{35}$ Deficiency of choline might genetically induce NAFLD by inducing irregular phospholipid synthesis, lipoprotein abnormalities and oxidative damage due to mitochondrial dysfunction. ${ }^{36}$ Thus, human studies are required to clarify its therapeutic role in NAFLD.

\section{Hepatoprotective agents}

TNF- $\alpha$ and has been shown to improve short term survival in severe alcoholic hepatitis and improves liver enzyme levels in NAFLD patients. Betaine, a methyl donor, protects against hepatic lipid accumulation, lowers aminotransferases and improves steatosis, inflammation and liver fibrosis. Angiotensin II promotes IR and hepatic fibrosis in animal models. Losartan, an angiotensin II receptor blocker, improved aminotransferases, serum markers of fibrosis and profibrotic cytokine in a pilot trial of seven subjects with NASH. Ursodeoxycholic acid
(UDCA), having anti-inflammatory, immune-modulating antiapoptotic properties, is widely used in chronic cholestatic liver diseases; but it is not recommended for the treatment of NAFLD. ${ }^{11}$

\section{Hypolipidemic drugs}

Anti-NASH drugs that target metabolic pathways aim to reduce the accumulation of fat in the liver including peroxisome proliferator-activated receptor (PPAR) agonists, e.g. pioglitazone, elafibranor, and saroglitazar; obeticholic acid (OCA) acting on farnesoid $\mathrm{X}$ receptor (FXR) that is involved in the metabolism of bile acids; and inhibitors of de novo fat synthesis, e.g. aramchol, acetyl coenzyme A carboxylase (ACC), e.g. GS-976 and incretin such as liraglutide. OCA is a bile acid that promotes insulin sensitivity and reduces triglycerides in the blood; $45 \%$ of patients with NAFLD had a significant improvement in their NAFLD activity score (NAS) and fibrosis. Resolution of NASH after treatment with OCA was not significantly different compared with placebo. ${ }^{6,7}$ A phase III trial evaluating efficacy and safety and of OCA in patients with compensated cirrhosis due to NASH ( registration no. NCT03439254) was started in 2017 and OCA is likely to be the first anti-NASH drug on the market. PPAR- $\alpha / \operatorname{PPAR}-\delta$ dual agonist, elafibranor (GFT-505), is also in phase III trial to evaluate its efficacy and safety in patients with NASH. Thyroid hormone has a vital role in lipid metabolism by activation of $\beta$-receptor, affecting serum cholesterol and TG levels and accumulation of fat in the liver. Thyroid hormone receptor $\beta$ selective to agonist, resmetirom (MGL-3196), is in phase III placebo-controlled study for evaluating its efficacy and safety in patients with NAFLD. Ianifibranor (IVA337), a new generation pan-PPAR agonist is in phase IIb trials from January 2017 to assess its effectiveness in the treatment of NASH. ${ }^{37}$

Aramchol, an arachidic acid-cholic acid complex (ACC), inhibits de novo lipid synthesis and reduces the hepatic fat content. It is in early clinical trials and phase IIb clinical trials for its therapeutic efficacy and safety in NASH patients with T2DM compared with placebo. Liraglutide, a glucagon-like peptide-1 (GLP-1) analogue relives NASH and reduces patient body weight; however, liver fibrosis was worsened in the liraglutide group. A third generation, long-acting GLP-1 analogue, semaglutide is under multicenter phase IIa trial to evaluate safety and efficacy compared to placebo in patients with NASH. Other agents such as ACC inhibitors, fibroblast growth factor-21 (FGF-21) analogue (BMS-986026), FGF-19 analogue NGM282 and PPAR$\alpha /$ PPAR-y dual agonist saroglitazar are phase IIa clinical trials in patients with NAFLD and NASH. ${ }^{37}$ Niacin beneficially affects NAFLD at the 3 major stages directly and, by affecting steatosis, it indirectly decreases the cascade effect on inflammation and fibrosis. It can be combined with other drugs for synergistic more intense and broader efficacy. In select patients, it may benefit frequently associated atherogenic dyslipidemia. ${ }^{38}$ 


\section{OXIDATIVE STRESS (OS), INFLAMMATION AND NAFLD}

Oxidative stress theory of ageing postulates age-related functional losses by the accumulation of ROS-induced damage. Liver function impairment and NAFLD are common among the elderly. Oxidative stress, lipotoxicity and inflammation play a vital role in the pathogenesis of NAFLD. There is a therapeutic potential of omega-3 polyunsaturated fatty acids (n-3-PUFA) mainly docosahexaenoic (DHA) and eicosapentaenoic (EPA) because of their antioxidant and anti-inflammatory properties. EPA and DHA serve as substrates for the formation of a novel series of specialised pro-resolving lipid mediators (SPMs). The SPMs include RvE-1 (Eseries resolvin derived from EPA), RvD1-6 (resolvins derived from DHA), protectins (NPD1, PDX) and maresin (MaR1-2). They have potent anti-inflammatory activity and pro-resolutive activity property at much lower doses than their n-3 PUFA precursors. ${ }^{39}$ Several studies in obese mice with IR and HS treated with some of these SPMs, including RvE1, RvD1, 17-HDHA, and maresin 1 decrease adipose tissue and liver inflammation and improved IR and reduce HS. Based on a systematic review of RCTs, regarding use of n-3 PUFA supplementation in NAFLD, these are an effective option to decrease liver fat and hepatic transaminases in adults with NAFLD. But their effectiveness in severe NAFLD or NASH is still unpredicted. It is difficult to define the dose of EPA or DHA that may be recommended for attenuation of NAFLD or NASH. Large-scale, well designed, RCTs are required to be conducted for a better understanding of the efficacy of n-3 PUFA in NAFLD/NASH treatment in adults. ${ }^{40}$

Other drugs that target oxidative stress (OS) or inflammation/injury induced by NASH include antioxidants, e.g. vitamin E, and those targeting apoptotic and TNF- $\alpha$ pathways, e.g. selonsertib, emricasan and pentoxyphyline as well as immunomodulators, e.g. amlexanox and cenicriviroc.

Selonsertib (GS-4997), apoptosis signal-regulate kinase-1 inhibitor, promotes inflammation and fibrosis during OS. It was found to improve hepatic fibrosis grading in patients with NASH in a phase II trial, and phase III trial is in progress. Emricasan is a caspase inhibitor that blocks liver cell apoptosis and inflammatory responses. It lowers serum levels of ALT and AST and CK-18 in patients with non-cirrhotic NAFLD. ${ }^{41}$ Ursodeoxycholic acid may improve acid adiponectin levels and IR, but does not affect NAFLD. Pentoxyphyline and probiotics require large scale randomised controlled trials for more evidence. $^{12}$ Degree of hepatic fibrosis (HF) is of great importance for the outcome of NASH. Simtuzumab (GS6624), an anti-lysyl oxidase like-2 drug, directly blocks the formation of collagen bands, thereby an antifibrotic effect. However, phase IIb trials evaluating the efficacy of simtuzumab in patients with hepatic fibrosis have been terminated prematurely. ${ }^{37}$

\section{GUT MICROBIOTA (GM)}

Obesity and MetS are associated with disturbance in gut microbiota. Microbiota induces higher extraction of energy from the diet and changes the lipid metabolism shifting it from oxidation to de novo production. ${ }^{42}$ Major phyla in the human gut are firmicutes (Lactobacillus, Peptoniphilus, Ruminococcus, Clostridium, and Eubacteria) and Bacteroidetes (Bacteroides, Prevotella); and other less abundant phyla include Actinobacteria (Bifidobacterium), Proteobacteria, and Verrumicrobia. ${ }^{43}$ However, the composition GM varies between individuals because of age, diet, infections, travel, gender, pregnancy, hormonal changes, and medication such as antibiotics and proton pump inhibitors. ${ }^{44}$

Dysbiota, an imbalance between healthy and diseasepromoting microorganisms, seems essential the development of NAFLD and NASH.D. It disrupts choline metabolism, which has been associated with NAFLD. Dysbiosis metabolise choline to trimethylamine (TMA) which gets further oxidized by hepatic monooxygenases to trimethylamine-N-oxide (TMAO). By this process; choline levels are reduced, TMAO levels are increased. Choline has a critical role in the synthesis of very low density lipoprotein (VLDL), and thus, it facilitates hepatic lipid exportation. Choline deficiency results in triglyceride accumulation in hepatocytes. A small human case study indicated that long-term, choline deficient, total parenteral nutrition (TPN) induced HS and the subjects resolved after receiving parenteral nutrition solutions containing choline chloride for 6 weeks. TMAO also increases IR, promotes inflammation and OS. A clinical study has demonstrated that higher serum levels of TMAO were associated with higher severity of NAFLD. ${ }^{45}$ In patients with NAFLD; there is bacterial overgrowth in the small intestine and increase intestinal permeability and thereby increasing endotoxin, lipopolysaccharide (LPS). TLR-4 is a receptor for LPS, from gram-negative bacteria, one of the most potent activators of Kuffer cells. Gut dysbiosis can also promote NASH by other mechanisms, e.g. ethanol production and disturbing choline metabolism. ${ }^{46}$ Agents for treatment of NAFLD regulate the enterohepatic circulation, including antiobesity drugs (e.g. orlistat), regulators of gut flora (e.g.IMM-124e) and solithromycin. Nevertheless, none of these has entered phase IIb or III clinical trials. ${ }^{37}$ The use of probiotics/synbiotics has been shown to improve the liver-specific markers of inflammation and steatosis in persons with NAFLD. More, well-designed, RCTs are needed to define the efficacy of probiotics/synbiotics for treatment of NAFLD. ${ }^{47}$

\section{COMBINATION THERAPY}

Increased physical activity, exercise and a low calorie diet should be advised to all the patients with NAFLD and NASH. Combination therapy targeting multiple stages of NASH may provide future direction for research. Combination therapy may have greater antifibrotic and 
anti-steatotic effects in comparison to monotherapy. A phase IIb trial was started in 2018 to evaluate combination therapy of selonsertib, ACC inhibitor GS-0976 and FXR agonist GS-9674 in NASH patients with bridging fibrosis or compensated cirrhosis and abnormal levels of hepatic biochemical indicators. ${ }^{37}$ In a clinical trial vitamin $\mathrm{E}$ in combination with vitamin $\mathrm{C}$ and atorvastatin is effective in reducing HS diagnosed NAFLD after 4 years of active treatment. ${ }^{48}$ Vitamin $\mathrm{E}$ has been combined with UDCA for long term ( $>2$ years) treatment showing improvement in most of the cases. ${ }^{49}$ Considering these observations, EASL and AASLD consider vitamin $\mathrm{E}$ as a potential short term treatment for non-diabetic adults with biopsy proven NASH. Until further supporting data in this regard is available, vitamin $\mathrm{E}$ is not recommended treatment of NASH patients with diabetes, NAFLD without liver biopsy, NASH cirrhosis, or cryptogenic cirrhosis. ${ }^{35}$

\section{CONCLUSION}

NAFLD is a common condition more prevalent, especially in the western world. It is a silent killer because mostly, there are no symptoms other than obesity. Currently, no drug has approval by the USFDA for prevention or treatment of NAFLD. Many targets for drug action have been explored, such as insulin sensitizers, hypolipidemic drugs and hepatoprotective agents and antioxidants. But most of them are unsafe on long term use. Further work is required in developing safe and effective medicine for NAFLD, because of its fatal outcome in the long run and requirement of liver transplantation in later stages of the liver disease which may not be available for everyone because of cost and resources. Thus, development of proper medication for treatment and prevention of NAFLD will reduce the global healthcare burden.

\section{Funding: No funding sources}

Conflict of interest: None declared

Ethical approval: Not required

\section{REFERENCES}

1. Younossi Z, Anstee QM, Marietti M, Hardy T, Henry L, Eslam M, et al. Global burden of NAFLD and NASH: trends, predictions, risk factors and prevention. Nat Rev Gastroenterol Hepatol. 2018;15 (1):11-20.

2. Chalasani N, Younossi Z, Lavine JE, Diehl AM, Blunt EM, Cusi K, et al. The diagnosis and management of non-alcoholic fatty liver disease: practice guideline by the american association for the study of liver diseases, American college of gastroenterology, and the American gastroenterological association. Hepatology. 2012;55 (6):2005-23.

3. Younossi ZM, Koenig AB, Abdelatif D, Fazel Y, Henry L, Wymer M. Global epidemiology of nonalcoholic fatty liver disease-Metanalytic assessment of prevalence, incidence and outcomes. Hepatol. 2016;64(1):73-84.

4. Hallsworth K, Adams LA. Lifestyle modification in NAFLD//NASH: facts and figures. JHEP Rep. 2019; 1(6):468-79.

5. Adams LA, Feldstein AE. Non-invasive diagnosis of non-alcoholic fatty liver and non-alcoholic steatohepatitis. J Dig Dis. 2011;12(1):10-6.

6. Shen FF, Li LG. Advances in noninvasive methods diagnosing nonalcoholic liver disease. J Dig Dis. 2016;17(9):565-71.

7. Wieckowska A, McCullough AJ, Feldstein AE. Noninvasive diagnosis and monitoring of non-alcoholic steatohepatitis: Present and future. Hepatol. 2007;46 (2):582-9.

8. Carter-Kent C, Zein NN, Feldstein AE. Cytokines in the pathogenesis of fatty liver and disease progression to steatohepatitis: implications for treatment. Am J Gastroenterol. 2008;103(4):1036-42.

9. Geroldi D, Falcone C, Emanuele E. Soluble receptors for advanced glycation end products from disease marker to potential therapeutic target. Curr Med Chem. 2006;13(17):1971-78.

10. Mikolasevic I, Milic S, Turk WT, Grgic I, Jakopcic I, Stimac D, Wensveen F, et al. Nonalcoholic fatty liver disease- A multisystem disease?. World J Gastroenterol. 2016;22(43):9488-505.

11. Adams LA, Angulo P. Treatment of non-alcoholic fatty liver disease. Postgrad Med J. 2006;82(967): 315-22.

12. Machado MV, Cortez-Pinto H. Non-alcoholic fatty liver disease: What the clinician needs to know. World J Gastroenterol. 2014;20(36):12956-80.

13. Moscatiello S, Di Luzio R, Bugianesi E, Suppini A, Hickman IJ, Di Domizio S, et al. Cognitivebehavioral treatment of non-alcoholic fatty liver disease: a propensity score-adjusted observational study. Obesity. 2011;19(4):763-70.

14. Takahashi H, Kotani K, Tanaka K, Egucih Y, Anzai $\mathrm{K}$, et al. Therapeutic approaches to non-alcoholic fatty liver disease: exercise intervention and related mechanisms. Front Endocrinol. 2018;9:588.

15. Zelber-Sagi Salomone F, Mlynarksky L. The Mediterranean dietary pattern as the diet of choice for non-alcoholic fatty liver disease: Evidence and plausible mechanisms. Liver Int. 2017;37:936-49.

16. European association for the study of the liver (EASL); European association for the study of diabetes (EASD); European association for the study of obesity (EASO). EAS-EASD-EASO clinical practice guidelines for the management of nonalcoholic fatty liver disease. J Hepatol. 2016;64:1388-402.

17. Dunn W, Sanyal AJ, Brunt EM, Unalp-Arida A, Donohue M, McCullough AJ, et al. Modest alcohol consumption is associated with decreased prevalence of steatohepatitis in patients with non-alcoholic fatty liver disease. J Hepatol. 2012;57(2):384-91.

18. Kwon HK, Greenson JK, Conjeevaram HS. Effect of lifetime alcohol consumption on the histological 
severity of non-alcoholic fatty liver disease. Liver Int. 2014;34:129-35.

19. Vuppalanchi R, Chalasani N. Nonalcoholic fatty liver disease and non-alcoholic steatohepatitis: selected practical issues in their evaluation and management. Hepatol. 2009;49(1):306-17.

20. Mazza A, Fruci B, Garinis GA, Giuliano S, Malaguarnera R, Belfiore A. The role of metformin in the management of NAFLD. Exp Diabetes Res. 2012;2012:716404.

21. Lin HZ, Yang SQ, Chuckaree C, Kuhajda F, Ronnet G, Diehl AM. Metformin reverses fatty liver disease in obese, leptin-deficient mice. Nat Med. 2000;6(9):998-1003.

22. Zhang X, Harmsen WS, Mettler TA Kim WR, Roberts RO, Therneau TM, et al. Continuation of metformin use after a diagnosis of cirrhosis significantly improves survival of patients with diabetes. Hepatol. 2014;60(6):2008-16.

23. Loomba R. Rationale for conducting a randomised trial to examine the efficacy of metformin in improving survival in cirrhosis: Pleiotropic effects hypothesis. Hepatol. 2014;60(6):1818-22.

24. Tacelli M, Celsa C, Magro B, Giannetti A, Pennisi G, Spatola F, et al. Antidiabetic drugs in NAFLD: the accomplishment of two goals at once? Pharmaceuticals. 2018;11:121.

25. Cooney RV, Harwood PJ, Franke AA, Narala K, Sundström AK, Berggren PO, et al. Products of gamma-tocopherol reaction with $\mathrm{NO} 2$ and their formation in rat insulinoma (RINm5F) cells. Free Radic Biol Med. 1995;19:259-69.

26. Christen S, Jiang Q, Shigenaga MK, Ames BN. Analysis of plasma tocopherols $\alpha, \gamma$, and 5-nitro- $\gamma$ in rats with inflammation by HPLC coulometric detection. J Lipid Res. 2002;43:1978-85.

27. Berbee M, Fu Q, Boerma M, Wang J, Kumar KS, Hauer-Jensen M. Gamma-tocotrienol ameliorates intestinal radiation injury and reduces vascular oxidative stress after total-body irradiation by an HMG-CoA reductase-dependent mechanism. Radiat Res. 2009;171:596-605.

28. Hadi HE, Vettor R, Rossato M. Vitamin E as a treatment for nonalcoholic fatty liver disease: reality or myth?. Antioxidants. 2018;7:12.

29. Miller ER, Pastor-Barriuso R, Dalal D, Riemersma RA, Appel LJ, Guallar E. Meta-analysis: Highdosage vitamin E supplementation may increase allcause mortality. Ann Intern Med. 2005;142:37-46.

30. Schurks M, Glynn RJ, Rist PM, Tzourio C, Kurth T. Effects of vitamin $\mathrm{E}$ on stroke subtypes: Metaanalysis of randomiszed controlled trials. BMJ. 2010;341:c5702.

31. Klein EA, Thompson IM, Tangen CM, Crowley JJ, Lucia MS, Goodman PJ, et al. Vitamin E and the risk of prostate cancer: the selenium and vitamin E cancer prevention trial (SELECT). JAMA. 2011;306:154956.

32. Zelber-Sagi S, Salomone F, Webb M, Lotan R, Yeshua H, Halpern Z, et al. Coffee consumption and non-alcoholic fatty liver onset: a prospective study in the general population. Transl Res. 2015;165:428-36.

33. Johnson S, Renwei WK, Govindarajan S, Yu MC, Yuan JM. Coffee consumption and reduced risk of hepatocellular carcinoma: Findings from the Singapore Chinese Health Study. Cancer Causes Control. 2011;22:503-10.

34. Chen S, Teoh NC, Chitturi S, Farrell GC. Coffee and non-alcoholic liver disease: brewing evidence from hepatoprotection. J Gastroenterol Hepatol. 2014;29 (3):435-41.

35. Perdomo CM, Fruhbeck G, Escalada J. Impact of nutritional changes on non-alcoholic fatty liver disease. Nutrtients. 2019;11:677.

36. Neuschwander-Teri BA, Loomba R, Sanyal AJ, Lavine JE, Van Natta ML, Abdelmalek MF, et al. Farnesoid $\mathrm{X}$ nuclear receptor ligand obeticholic acid for non-cirrhotic, non-alcoholic steatohepatitis (FLINT): a multicentre, randomised, placebocontrolled trial. Lancet. 2015;385(9972):956-65.

37. Tang JT, Mao YM. Development of new drugs for the treatment of non-alcoholic steatohepatitis. J Dig Dis. 2020;21:3-11.

38. Kashyap ML, Ganji S, Nakra NK, Kamanna VS. Niacin for treatment of non-alcoholic fatty liver disease (NAFLD): novel use for an old drug? J Clin Lipidol. 2019;13(6):873-79.

39. Martinez-Fernandez L, Laiglesia LM, Huerta AE, Martínez JA, Moreno-Aliaga MJ. Omega-3 fatty acids and adipose tissue function in obesity and metabolic syndrome. Prostaglandins Other Lipid Mediat. 2015;121(Part A):24-41.

40. Yang J, Fernandez-Galilea M, Martinez-Fernandez L, González-Muniesa P, Pérez-Chávez A, Martínez JA, et al. Oxidative stress and non-alcoholic fatty liver disease: effects of omega-3 fatty acid supplementation. Nutrients. 2019;11(4):872.

41. Shiffman M, Freilich B, Vuppalanchi R, Watt K, Chan JL, Spada A, et al. Randomised clinical trial: emricasan versus placebo significantly decreases ALT and caspase 3/7 activation in subjects with nonalcoholic fatty liver disease. Aliment Pharmacol Ther. 2019;49(1):64-73.

42. Machado M V, Cortez-Pinto H. Gut microbiota and non-alcoholic fatty liver disease. Ann Heptatol. 2012;11(4):440-9.

43. Tap J, Mondot S, Levenez F, Pelletier E, Caron C, Furet JP, et al. Towards the human intestinal microbiota phylogenetic core. Env Microbiol. 2009;11(10):2574-84.

44. Xie C, Halegoua-DeMarzio D. Role of probiotics in non-alcoholic fatty liver disease: does gut microbiota matter?. Nutrients. 2019;11(11):2837.

45. Chen YM, Liu Y, Zhou RF, Chen XL, Wang C, Tan $\mathrm{XY}$, et al. Associations of gut-flora-dependent metabolite trimethylamine-N-oxide, betaine and choline with non-alcoholic fatty liver disease in adults. Sci Rep. 2016;6:19076.

46. Buchman AL, Dubin MD, Moukarzel AA, Jenden DJ, Roch M, Rice KM, et al. Choline deficiency: A 
cause of hepatic steatosis during parenteral nutrition that can be reversed with intravenous choline supplementation. Hepatol. 1995;22(5):1399-403.

47. Sangouni AA, Ghavamzadeh S. A review of synbiotic efficacy in non-alcoholic fatty liver disease as a therapeutic approach. Diabetes Metab Syndr. 2019;13(5):2917-22.

48. Sharpton SR, Maraj B, Harding-Theobald E, Vittinghoff E, Terrault NA. Gut microbiometargeted therapies in non-alcoholic fatty liver disease: a systematic review, meta-analysis, and metaregression. Am J Clin Nutr. 2019;110(1):139-49.
49. Foster T, Budoff MJ, Saab S, Ahmadi N, Gordon C, Guerci AD. Atorvastatin and antioxidants for the treatment of non-alcoholic fatty liver disease: The St Francis Heart Study randomised clinical trial. Am J Gastroenterol. 2011;106(1):71-7.

Cite this article as: Braich JS. Potential targets for prevention and treatment of non-alcoholic fatty liver disease in adults. Int $\mathbf{J}$ Basic Clin Pharmacol 2020;9:1922-30. 